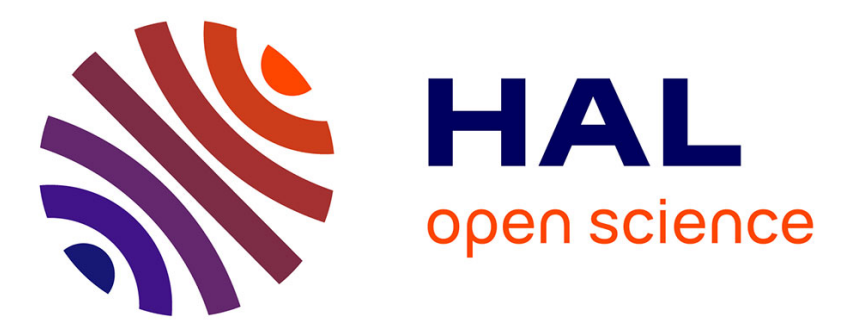

\title{
Growth and molecular responses to long-distance stimuli in poplars: bending vs flame wounding
} Aude Tixier, Eric Badel, Jérôme Franchel, Wassim Lakhal, Nathalie Leblanc-Fournier, Bruno Moulia, Jean-Louis J.-L. Julien

\section{- To cite this version:}

Aude Tixier, Eric Badel, Jérôme Franchel, Wassim Lakhal, Nathalie Leblanc-Fournier, et al.. Growth and molecular responses to long-distance stimuli in poplars: bending vs flame wounding. Physiologia Plantarum, 2014, 150 (2), pp.225-237. 10.1111/ppl.12089 . hal-00964793

\section{HAL Id: hal-00964793 https://hal.science/hal-00964793}

Submitted on 16 Mar 2018

HAL is a multi-disciplinary open access archive for the deposit and dissemination of scientific research documents, whether they are published or not. The documents may come from teaching and research institutions in France or abroad, or from public or private research centers.
L'archive ouverte pluridisciplinaire HAL, est destinée au dépôt et à la diffusion de documents scientifiques de niveau recherche, publiés ou non, émanant des établissements d'enseignement et de recherche français ou étrangers, des laboratoires publics ou privés. 


\title{
Growth and molecular responses to long-distance stimuli in poplars: bending vs flame wounding
}

\author{
Aude Tixier ${ }^{a, b}$, Eric Badel ${ }^{a, b}$, Jerome Franchel ${ }^{a, b}$, Wassim Lakhal ${ }^{a, b}$, Nathalie Leblanc-Fourniera,b, \\ Bruno Moulia ${ }^{a, b}$ and Jean-Louis Julien ${ }^{a, b, *}$ \\ aClermont Université, Université Blaise-Pascal, UMR547 PIAF, BP 10448, 63000 Clermont-Ferrand, France \\ IINRA, UMR547 PIAF, 63100 Clermont-Ferrand, France
}

doi:10.1111/ppl.12089

\begin{abstract}
Inter-organ communication is essential for plants to coordinate development and acclimate to mechanical environmental fluctuations. The aim of this study was to investigate long-distance signaling in trees. We compared on young poplars the short-term effects of local flame wounding and of local stem bending for two distal responses: (1) stem primary growth and (2) the expression of mechanoresponsive genes in stem apices. We developed a non-contact measurement method based on the analysis of apex images in order to measure the primary growth of poplars. The results showed a phased stem elongation with alternating nocturnal circumnutation phases and diurnal growth arrest phases in Populus tremula $\times$ alba clone INRA 7171B4. We applied real-time polymerase chain reaction (RT-PCR) amplifications in order to evaluate the PtaZFP2, PtaTCH2, PtaTCH4, PtaACS6 and PtaJAZ5 expressions. The flame wounding inhibited primary growth and triggered remote molecular responses. Flame wounding induced significant changes in stem elongation phases, coupled with inhibition of circumnutation. However, the circadian rhythm of phases remained unaltered and the treated plants were always phased with control plants during the days following the stress. For bent plants, the stimulated region of the stem showed an increased PtaJAZ5 expression, suggesting the jasmonates may be involved in local responses to bending. No significant remote responses to bending were observed.
\end{abstract}

\section{Introduction}

Under natural conditions, plants experience a daily mechanically challenging environment coupling internal mechanical stresses such as self-loading (Telewski 2006), internal growth, and eventually fruit loading (Almeras et al. 2004). Adding to these internal stimulations, a fluctuating external environment also shapes plant development. Wind is the most important external force, with multi-component factors (frequency, intensity, and duration) leading to mechanical deformations of plant organs that increase with increasing plant height and leaf area (James et al. 2006). It has been demonstrated that organ deformation is a key driver of morphological responses (Moulia et al. 2011). The intensity of the response is a function of the sum of strains experienced by the plant (Martin et al. 2010). The morphological responses of plants to these mechanical deformations are called thigmomorphogenesis (Jaffe 1976, Jaffe et al. 1984). These responses have been studied in gymnosperms (Telewski and Jaffe 1986) and angiosperms

Abbreviations - ACS, aminocyclopropane-carboxylate synthase; ANOVA, analysis of variance; JAZ, jasmonate ZIM-domain; LSM, least-square minimization; PIN, protease inhibitor genes; qPCR, quantitative polymerase chain reaction; RT-PCR, real-time polymerase chain reaction; $\mathrm{TCH}$, touch; ZFP, zinc finger protein. 
(Telewski and Pruyn 1998, Doaré et al. 2004, Braam 2005, Kern et al. 2005) in works that mainly focused on long-term effects of wind. Thigmomorphogenesis generally leads to inhibited primary elongation and increased radial growth (Telewski and Pruyn 1998). Growth responses are coordinated with biomass reallocation, leaf loss, conductance variations, phloem transport block (Telewski 2006), flowering time, dormancy, chlorophyll content and drought resistance (Braam 2005).

Inter-organ communication is essential for plants to coordinate development and acclimate it to fluctuant environment (Zimmermann et al. 2009). Such coordinated responses highlight the extent of long-distance signaling mechanisms in plants. Understanding longdistance signaling is a major scientific challenge, not least owing to the multiplicity of signals and stimuli involved. Although some signals are volatile, like ethylene (Zimmermann et al. 2009), methyl salicylate and methyl jasmonates (Heil and Ton 2008), other studies in the literature report that other transmissions should mainly occur through phloem and xylem (Fromm and Lautner 2007, Neumann 2007, Zimmermann et al. 2009). Heil and Ton (2008) reviewed the combination of vascular and airborne signals for plant defense. The authors suggested that the combination of those signaling pathways provided option for a fine-tuning and balanced systemic resistance. Vascular chemical, hydraulic or electrical signals have been hypothesized or reported (Davies 1987, Malone 1996, Christmann et al. 2007). In phloem, chemical signals such as metabolites, phytohormones, sugars, reactive oxygen species, proteins (Dinant 2008) and RNA have been identified (Kehr and Buhtz 2008), and also phytohormones such as auxin, gibberellins, cytokinins, salicylic acid, abscisic acid and jasmonates have been found (Hoad 1995, Neumann 2007, Dinant 2008). Phloem is also the site of electrical signaling with typical velocity of $1 \mathrm{~cm} \mathrm{~s}^{-1}$, known as action potential (Julien and Frachisse 1992). Finally external mechanical stresses trigger a reduction in phloem transport velocity (Jaeger et al. 1988). Xylem is also involved in long-distance signaling as its structure enables rapid transport of chemical signals (Malone 1996), like ethylene, abscisic acid and protein (Neumann 2007). Xylem also carries electrical signaling: indeed a wounding stimulus generates a slow wave, also called variation potential (Stankovic and Davies 1998). Slow wave is an electrical signal transmitted over long distance through the xylem with a typical velocity of $1 \mathrm{~mm} \mathrm{~s}^{-1}$ (Julien and Frachisse-Stoiljkovic 1994). A xylemian hydraulic signal has been also reported, generated by a pressure variation and transmitted through a vascular continuum (Malone et al. 1994). Slow wave is thought to be associated to the hydraulic signal
(Stahlberg et al. 2005). Indeed when Stahlberg et al. (2005) excised roots to induce a wounding signal in Helianthus annuus, they found that the range of slow wave was considerably shortened when root excision was carried out in air rather than in water. The open ends of the cut vessels were quickly filled with air so that the developing surface tension quickly restored the initial xylem tension; thus limiting the conduction of a change in hydraulic pressure.

In contrast with plant systemic defense to biotic aggressions, the molecular processes governing responses to thigmomorphogenesis and wounding have mainly been studied locally (i.e. at the site of the stimulation) (Braam and Davis 1990, Heil and Ton 2008). The Touch $(T C H)$ genes are upregulated in responses to touch stimuli and were isolated from Arabidopsis thaliana seedlings (Braam and Davis 1990). Long term exposure to wind of poplars revealed changes in expression of several genes involved in signaling pathways such as kinases, calmodulin and membrane proteins. Later, Martin et al. (2009) showed the involvement of the genes PtaZFP2 (zinc finger putative transcription factor), PtaTCH2 (calmodulin-like protein) and PtaACS6) (acid 1 -aminocyclopropane-1-carboxylate synthase) in the early stages of mechanoperception in poplars. Plants also response to wounding with upregulation of the WIN genes (wound inducible) (Reymond and Farmer 1998). Touch and wound responses share the expression of some genes, suggesting that they share some mechanical signaling component (Reymond et al. 2000). Besides Heil et al. (2012) studied differences in transcriptional responses between mechanical wounding and flame wounding. These results suggest that several components of external stimuli are perceived and that molecular responses to those stimuli are balanced and do not behave on all-or-nothing basis. The molecular processes governing remote responses have mainly been studied in response to localized wounding. Systemic proteinase inhibitors accumulation in tomato leaves has been identified $20 \mathrm{~min}$ after localized wounding (Farmer et al. 1992); this has been recognized also in tobacco and poplar (Malone 1996). Heil et al. (2012) showed high similarities in transcriptional changes after jasmonates (JA) application and flame wounding, suggesting that global changes in gene expression after flame wounding were mediated via JA. Other studies report that JA are involved in distal responses to wounding: Zhang and Turner (2008) presented evidence for the role of JA in growth responses to wounding in A. thaliana and showed that wounding reduced the growth of wild-type plants but not of mutants that were unable to synthesis or respond to JA. Glauser et al. (2009) estimated a $3.4-4.5 \mathrm{~cm} \mathrm{~min}^{-1}$ velocity of propagation 
of the signal leading to systemic JA accumulation. Using quantitative liquid chromatography-mass spectrometry on flash-frozen tissues, they found that JA accumulated in $30 \mathrm{~s}$ in wounded leaves and in $120 \mathrm{~s}$ in distal leaves. Wasternack et al. (2006) reviewed studies on tomatoes grafted with JA-deficient and JA-insensitive mutants. The generation of a systemic signal required local JA biosynthesis and JA perception in the systemic leaf, but systemic JA biosynthesis was not necessary.

Long-distance signaling and remote responses also occur in thigmomorphogenesis (Erner et al. 1980). In order to understand thigmomorphogenesis mechanisms, experiments based on rubbing, touching and bending have been developed (Jaffe 1976, Erner et al. 1980, Coutand and Moulia 2000, Pruyn et al. 2000). Inhibition of primary growth response has been studied as a long-term response and mainly done on herbaceous species such as maize, barley, bean, sunflower and tomato (Coutand 2010). To our knowledge, no study has addressed the short-term primary growth response to distal perturbation in tree species. Employing a biomechanical approach, Coutand and Moulia (2000) showed that a single bending of a tomato stem generates a cessation of growth within $5 \mathrm{~min}$ and that lasted for several hours. Martin et al. (2010) observed local effects of bending on secondary growth for poplar (with both short term and very long terms, components lasting several days), but the primary growth responses remain unclear. The aim of this study was to investigate long-distance signaling in trees. We compared the short-term effects of local flame wounding - i.e. a large multicomponents wounding stress but non-controlled-and of local stem bending - i.e. a controlled mechanical stimulus. Two distal responses were studied: (1) the stem primary growth and (2) the expression of mechanoresponsive genes in stem apices. Poplar was used owing to its fast growth and the availability of molecular tools. On the one hand, we developed a non-contact measurement method, based on the analysis of apex images, in order to measure the stem primary growth while avoiding mechanical perturbations of the plants during experiments. On the other hand, real-time quantitative polymerase chain reaction (RT-qPCR) was used to study the expression in the apical region of mechanoresponsive genes involved in several signaling pathways.

\section{Materials and methods}

\section{Plant material and culture conditions}

Young poplars (Populus tremula $\times$ alba, clone INRA 717-1B4) were propagated clonally by in vitro micropropagation on Murashige and Skoog medium
(Murashige and Skoog 1962). Once they reached a height of about $40 \mathrm{~mm}$, they were gradually acclimatized (Martin et al. 2009) on a hydroponic solution (Morizet and Mingeau 1976) through decreasing relative humidity. Trees were then placed in a growth chamber $\left(16 \mathrm{~h} / 8 \mathrm{~h}\right.$ light/dark cycle at $40 \mu \mathrm{mol} \mathrm{m}^{-2} \mathrm{~s}^{-1}$ and $22^{\circ} \mathrm{C} / 18{ }^{\circ} \mathrm{C}$ with air relative humidity of $60 \%$ ). Two months after micropropagation, the poplars were ready for experiments: at this stage, stems were about $450 \mathrm{~mm}$ $( \pm 4.3 \mathrm{SE})$ high and $4.5 \mathrm{~mm}( \pm 0.8 \mathrm{SE})$ in diameter.

\section{Local external stresses}

Plants were transferred to another tank where they experienced the stress treatment. However, moving induces mechanical stimuli which stimulates plant acclimation and decreases their molecular responses (Martin et al. 2010) and phloem transport velocity (Jaeger et al. 1988). Thus, the plants were installed in the new tank 4 days before we applied the stress in order to avoid impact of the displacement in the subsequent experiments. The stems were attached on a vertical metal support with individual clamping rings (Fig. 1A). A foam layer was rolled around each stem to avoid stem wounds. Plants were grown hydroponically in a nutrient solution that was oxygenated by four aquarium pumps. First basal leaves were removed from the stem in order to avoid uncontrolled mechanical stimuli during treatments. The stimuli were applied at 10:00 h. for all experiments.

A basal segment of the stem was manually bent during $8 \mathrm{~s}$ by moving it along a plastic circular tube (Martin et al. 2009). The end angle was $\pi / 4$. The constant radius of curvature allowed the strain field in the stem to be controlled and constant throughout the bent stem segment (Moulia et al. 2011). The average sum of strains applied to stem segment was evaluated at $13.2 \mathrm{~mm}^{3}$ $( \pm 1.52 \mathrm{SE})$. These sums of strains were high enough to induce local secondary growth and molecular responses yet low enough to avoid wounding (Martin et al. 2009).

Flame wounding was performed on the total surface of the most basal leaf, until the tissues withered. Flamed leaves were kept on plants during all experiments.

\section{Primary stem elongation measurement, image processing, curve treatments}

Daily plant height was measured by hand with a ruler at a fixed time $(10: 00 \mathrm{~h})$ on a dataset of three repetitions of 20 plants: 7 plants were flamed (one basal leaf), 7 were bent (stem segment), and 6 were unchallenged control plants. Daily growth was measured during 2 days before treatments and 3 days after. The daily growth of day $D$ was measured on $\mathrm{D}+1$. 


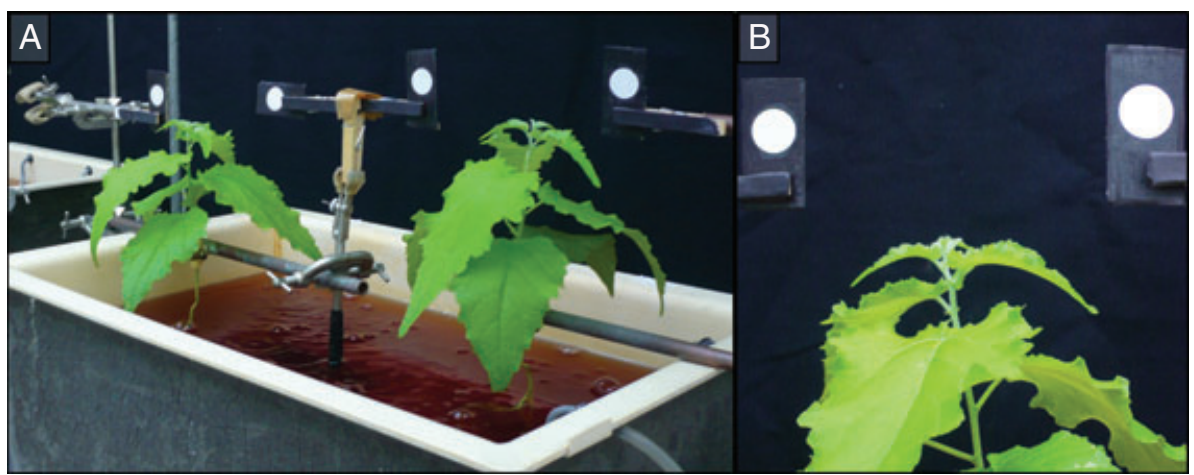

Fig. 1. Device for primary stem elongation measurement and bending treatments. (A) Plants were vertically aligned upright along a metal support with roots in a hydroponic solution. (B) Apex images were recorded every 15 min during 5 days. Two circular white reference patterns were used to take possible camera movements into account.

For short-term growth response, stem height was measured continuously using digital cameras. An image of each apical part was recorded every 15 min during the 5 days of the experiment (Fig. 1A). This non-contact method avoided mechanical perturbations of the plants during experiments. A similar method of measurement was pioneered by Jaffe et al. (1985) with a non-intrusive auxonometric system. As green-light wavelengths are not perceived by plants (Richter and Fukshansky 1994), green filters were fixed in front of the camera flashes in order to avoid light-plant interactions during the night. Shutter speed was tuned to the minimum with forced flash to ensure the same quality of pictures for day and night exposures.

Two circular white patterns were installed on the left and right sides of the plant within the frame of the image (Fig. 1B). These patterns were used as positioning references to take into account any translational and rotational camera movements, and one of the patterns was also used for scale calibration purposes. An automatic procedure was developed to isolate, threshold (applying Otsu's method; Otsu 1979) and label these patterns in the image. Finally, the position of the center of mass of the circular pattern was computed for each image. The pattern trajectories were used to correct the position of the apex.

The tracking of apex position in each picture made possible to plot stem elongation curves in a twodimensional space. Curves were then processed by segmented regression using MATLAB software and the SLM tool package ( $D^{\prime}$ Errico 2009). The diurnal growth arrest phases showed linear patterns while nocturnal circumnutation phases showed sinusoidal patterns. Vertical apex movements can be modeled as the sum of a linear growth and a sinusoidal function:

$$
y=\underbrace{(\mathrm{at}+\mathrm{b})}_{\text {linear growth }}+\underbrace{\mathrm{c} \sin (\omega \mathrm{t}+\mathrm{d})}_{\text {circumnutation }}
$$

where $y$ is the vertical coordinate of the apex of the shoot, $\mathrm{t}$ is the time, $\mathrm{a}$ is the growth rate, and $\mathrm{c}$ and $\omega$ are the magnitude and angular frequency (in rad $\mathrm{min}^{-1}$ ) of the circumnutation, respectively (c and d are constants that are dependent on initial conditions). The first term refers to the intrinsic growth of the apical part, whereas the second term refers to lateral movements due to circumnutation. Stem elongation curves were partitioned into irregular intervals, and linear regression was adjusted for each interval. The limits of the segments were determined by least-square minimization (LSM) by successive iterations (D'Errico 2009). The reliability of segmented regressions on sinusoidal curves of circumnutation phases was assessed by comparing it with linear regression between the maximum and sinusoidal regression (data not shown). The individual lengths of the elongation curve segments correspond to one phase duration $(\mathrm{min})$ and their slope corresponds to the growth rate $\left(\mathrm{mm} \mathrm{min}^{-1}\right)$.

\section{RNA isolation and CDNA synthesis}

Total RNA was extracted from the apex of control plants and at 1, 2, 3 and $24 \mathrm{~h}$ after flame wounding the basal leaf or at $0.5,1,2,3,5,18$ and $24 \mathrm{~h}$ after bending the basal part of the stem. Relative transcript accumulation was determined by real-time qPCR and corresponds to means of two independent experiments. Total RNA was extracted from apices using hexadecyl-trimethylammonium bromide extraction buffer as described in Chang et al. (1993), then treated with RNasefree RQ1 DNase (Promega, Charbonnières-les-Bains, 
Table 1. Gene primers sequences and properties. Tm: annealing temperature, S: sense primer, AS: antisense primer.

\begin{tabular}{|c|c|c|c|c|}
\hline Genes & $\operatorname{Tm}\left({ }^{\circ} \mathrm{C}\right)$ & Amplicon length $(\mathrm{pb})$ & Function & Primer sequence \\
\hline PtaZFP2 & 61 & 287 & Zinc-finger putative transcription factor & $\begin{array}{l}S->\text { CGTGCGAGTAAGAAACC } \\
\text { AS -> CACAGAACTCTCTTGCGTCT }\end{array}$ \\
\hline PtaACS6 & $56-60$ & 454 & ACC synthase (ethylene biosynthesis) & $\begin{array}{l}\mathrm{S}->\text { ACTACAGGAAGATATTGAATG } \\
\text { AS -> TGGCTTCGAGCAATGAAAAGA }\end{array}$ \\
\hline PtaTCH4 & $58-62$ & 283 & Xyloglucan endotransglycosylase & $\begin{array}{l}\text { S -> GATGAGATAGATTTTGAATTC } \\
\text { AS -> ATCAGCATTCCATAGACTCGA }\end{array}$ \\
\hline PtaTCH2 & 61 & 243 & Calmodulin like protein, $\mathrm{Ca}^{2+}$ binding prot & $\begin{array}{l}S->\text { TGATCAAGATGGTGATGGTAATG } \\
\text { AS -> CGCAAAAACATCAATGGAAA }\end{array}$ \\
\hline PtaJAZ5 & 58 & 246 & Jasmonate zim domain protein 5 & $\begin{array}{l}\text { S -> TCTTGCTGCAACCCTTTCTT } \\
\text { AS -> GGTAAGGCCGAGGAGATCAT }\end{array}$ \\
\hline PtaUBC & 60 & 146 & Ubiquitin & $\begin{array}{l}\text { S -> CCCGGCTCTAACCATATCCA } \\
\text { AS -> GGGTCCAGCTTCTTGCAGTC }\end{array}$ \\
\hline
\end{tabular}

France). RNA quantity and quality were checked spectrophotometrically using a NanoDrop apparatus (Thermo Fisher Scientific Inc., Waltham, MA) and counter-checked by agarose gel electrophoresis. cDNA was synthesized by reverse transcription from $1 \mu \mathrm{g}$ of total RNA using oligo(dT) and SuperScript III enzyme (Invitrogen, Cergy Pontoise, France), according to the manufacturer's instructions.

\section{Real-time quantitative RT-PCR experiments}

Real-time polymerase chain reaction (RT-PCR) amplifications were performed on an iCycler IQ (Bio-Rad Laboratories, Hercules, CA) with SYBR Green as fluorescent dye, as in Martin et al. (2009). Each PCR reaction $(25 \mu \mathrm{l})$ contained the following: cDNA $(4 \mu \mathrm{l}$ of 1:20 dilution of the first cDNA strands), $12.5 \mu$ l MasterMix (Mesa green qPCR MasterMix Plus, Eurogentec, Seraing, Belgium), and specific primers (0.2 $\mu \mathrm{M}$ each). PCR started with a heat step at $94{ }^{\circ} \mathrm{C}$ for $5 \mathrm{~min}$ and then the following PCR cycling program: 40 cycles of denaturation $\left(95^{\circ} \mathrm{C}\right.$, $15 \mathrm{~s})$, annealing ( $\mathrm{Tm}$, Table 1$)$, and elongation $\left(72^{\circ} \mathrm{C}\right.$, $20 \mathrm{~s}$ ), ending with a final elongation step at $72{ }^{\circ} \mathrm{C}$ for 5 min. PtaZFP2, PtaTCH2, PtaTCH4, PtaACS6 and PtaJAZ5 transcripts were amplified using specific primers (Table 1).

Relative quantitative abundances of these target gene transcripts were calculated by comparison against the expression of the reference gene PtaUBC, coding for ubiquitin, using the delta-delta method mathematical model (McMaugh and Lyon 2003):

$$
\mathrm{Qr}=2^{\left(\mathrm{C}_{\text {control }}-\mathrm{C}_{\text {treated }}\right)} \text { (target-reference) }
$$

where $\mathrm{C}$ is the number of PCR cycles required to reach the quantitative detection threshold. The specificity of the primer-driven sequence amplification was validated by determining the melt curves for the PCR products at the end of each run. Sequence data from this article can be found in the GenBank/EMBL data library.

\section{Statistical analysis}

Growth phases data were analyzed by two-way analysis of variance (ANOVA) followed by a post hoc Tukey's HSD test to detect significant effect of days and treatments. Results were considered statistically different at $P<0.05$. Daily longitudinal growth data did not show normality and homogeneity of variance. Thus, the non-parametric test of Kruskal and Wallis and Kruskal-Wallis multiple comparisons were used to detect differences between days for each treatment.

As empirical errors in $\mathrm{Qr}$ increased with $\mathrm{Qr}$ values, consistently with the above exponential formulation, statistical processing was performed on log-transformed data. Qr was then analyzed by ANOva followed by a post hoc Tukey's HSD test to detect significant effect of time on $\mathrm{Qr}$ values. Results were considered statistically different at $P<0.05$.

\section{Results}

\section{Poplars show a phased stem elongation with nocturnal circumnutation phases and diurnal growth arrest phases}

Stem elongation curves obtained by the analysis of apex images revealed that poplar showed a phased stem elongation with alternating nocturnal circumnutation phases and diurnal growth arrest phases (Fig. 2A). Diurnal growth arrest phases (from around 06:00 $\mathrm{h}$ to $18: 00 \mathrm{~h}$ ) were characterized by small slopes whereas nocturnal circumnutation phases (from around 18:00 h to 06:00) were characterized by helical movements of the apex together with intense growth. Poplar circumnutation showed pendulum-like 

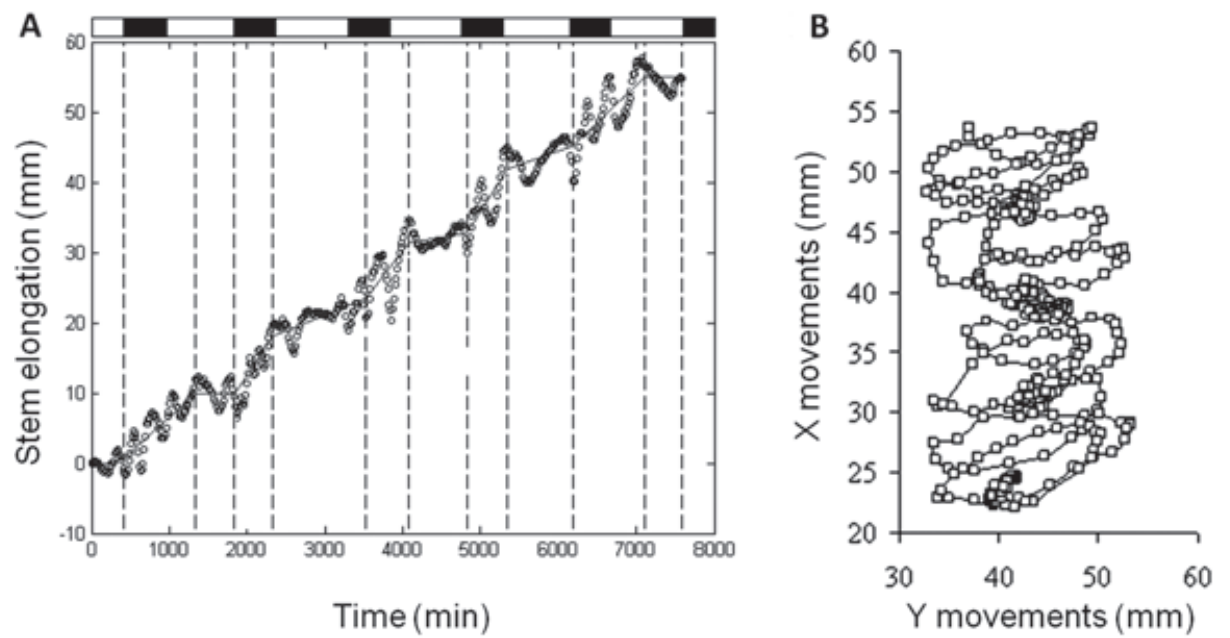

Fig. 2. Poplar phased stem elongation processing with automated segmented regression. Stem elongation of one plant (A) is expressed in millimeter as a function of time. Poplar stem elongation alternates between nocturnal circumnutation phases and diurnal growth arrest phases. Photoperiod is represented by black (light off) and white (light on) bars. Stem elongation fits were processed using automated segmented regression with matLaв Software using the SLM toolbox. The iterative process provided a linear fit for each period and defined start and end points (vertical dotted lines) by minimizing the sum square (SS). Plain lines show output of segmented regressions. Stem elongation curves were then characterized by several segments where lengths correspond to phase duration $(\mathrm{min})$ and slopes correspond to growth rates $\left(\mathrm{mm} \mathrm{min}^{-1}\right)$ during growth phases. (B) the $2 \mathrm{D}$ circumnutation pendulum-like movements of a circumnutation phase expressed in millimeter for one plant.

rotations. Fig. 2B shows typical two-dimensional (2D) nutation movements during 4 days for one plant. Each circumnutation phase involved two to four nutation cycles lasting $3-6 \mathrm{~h}$. The magnitude of lateral nutation movements ranged between 2 and $20 \mathrm{~mm}$. This high range of magnitude in circumnutation can have a strong impact on growth measurements, as discussed further on.

\section{Treatments affect stem elongation phases but not its circadian rhythm}

Fig. 3 shows the time-course evolution of daily longitudinal growth of control plants compared to daily growth after bending the basal part of the stem or flame wounding a basal leaf. Mean poplar growth was $13.1 \mathrm{~mm}$ ( $\pm 0.3 \mathrm{SE})$ per day and remained fairly stable during the 6 days of the experiments for control plants. However, flamed plants showed a strong reduction in daily growth during the first day of treatment; down to $5.8 \mathrm{~mm}( \pm 0.5$ $\mathrm{SE})$ per day. Bending, on the contrary, had no significant effect on daily growth during the day of treatment (Dday), although we observed a slight decrease in daily growth the day after bending ( $10.3 \mathrm{~mm} \pm 0.6 \mathrm{SE})$ per day on $\mathrm{D}+1$ on bent plant).

Using the images of apex and the corresponding displacement curves for the apex (Fig. 2A) made it possible to measure growth responses to treatments (Fig. 4) with a better time resolution. Growth phase duration (min) and growth rate $\left(\mathrm{mm} \mathrm{min}^{-1}\right)$ were compared between treatments. Fig. 4A shows comparisons of diurnal growth arrest durations between controls, flamed and bent plants from the treatment time until 2 days posttreatment. Phase durations are expressed in minutes, and data represent means of three independent experiments. During the day when treatments were applied, flame wounding of the basal leaf induced an extension of the duration of the phase of growth arrest by a factor of three. Control plants showed diurnal growth arrest phases that lasted 517 min $( \pm 32 \mathrm{SE})$, while flamed plants showed longer diurnal growth arrest phases that lasted $1615 \mathrm{~min}( \pm 347$ SE) during the day when treatment was applied. In some cases, the diurnal growth arrest phase extended to the day after treatment and merged with it. In this case the nocturnal circumnutation phase was inhibited and there was a drastic reduction of the relative growth over the course of the night following the treatment (Fig. 4B).

Fig. 4B compares relative nocturnal growth between controls, flamed and bent plants from 1 day before treatment to 2 days posttreatment. Each plant's nocturnal growth was normalized by that of the night before the treatment $(D-1)$. Control plants presented a regular linear increase in nocturnal relative growth, whereas both treatments induced a reduction in relative nocturnal growth the night following treatment (flamed plants: $P<0.01$, bent plants: $P=0.053$ ).

Two days after the treatments, poplar growth phase durations and relative growth rate returned to control 

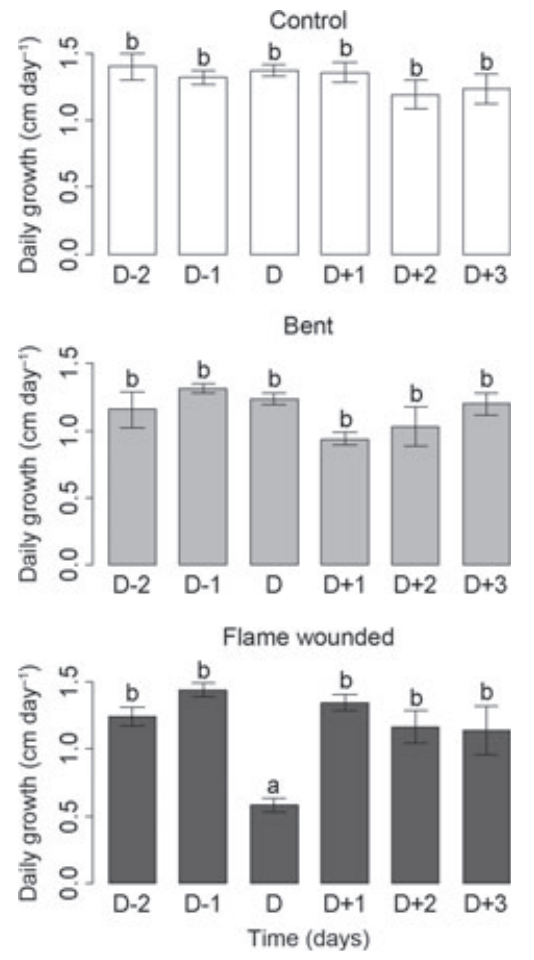

Fig. 3. Time-course evolution of daily primary growth of poplar stems. Control plants (white bars) and plants subjected to a stem bending (gray bars) or leaf flame wounding (black bars) were measured manually. Treatments were applied on D-day. Each bar represents mean values of three repeated experiments of 20 plants each. Error bars represent standard error (sE). Bendings were applied using $40 \mathrm{~mm}$ diameter tubes for an average sum of strains of $13.2( \pm 1.52 \mathrm{sE}) \mathrm{mm}^{3}$. Mean daily growths were compared using Kruskal-Wallis one-way Anova. Letters indicate significantly different groups (Kruskal-Wallis multiple comparisons, $P<0.05)$.

levels. While significant changes were induced on stem elongation phases, the circadian rhythm of phases remained unaltered and the treated plants were always phased with control plants the days after the treatments (data not shown).

\section{Molecular markers of mechanoperception are regulated over long-distance after flame wounding}

To prospect the molecular responses to the treatments, the expression of mechanoresponsive genes was studied locally and in apices using qPCR. Molecular responses to bending were first studied locally. Fig. 5 shows that the accumulation of PtJAZ5, a jasmonate sensitive gene, mRNA started $30 \mathrm{~min}$ after bending. Peak transcript accumulation was reached $1 \mathrm{~h}$ after bending with a 25fold increase in relative transcript abundance. Moreover, a slight but significant accumulation of transcripts could still be observed $3 \mathrm{~h}$ after bending. Expression level
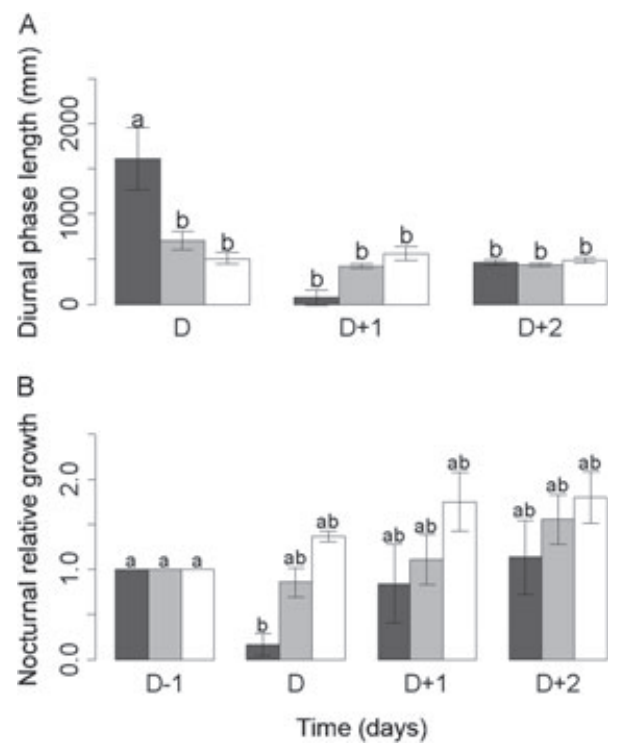

Fig. 4. Effect of treatments on stem elongation phases. Treatments were: one basal leaf was flamed (black bars) or a basal stem segment was bent (gray bars). White bars represent the control plants. Stem elongation curves were processed with segmented regression as described in the text. Growth was characterized by diurnal growth arrest phase duration (min) and nocturnal growth rate $\left(\mathrm{mm} \mathrm{min}^{-1}\right)$. Nocturnal growth rates were normalized to the growth rate of the night preceding treatment. Results are means of three independent experiments. Error bars represent standard error (SE). The raw data was analyzed by ANova followed by a post hoc Tukey's HSD test to detect significant differences at $P<0.05$. Letters indicate significantly different groups (Tukey's HSD test, $P<0.05$ ).

returned to control levels $5 \mathrm{~h}$ after stem bending. This result demonstrates that the plants had locally perceived the mechanical stimulus. Then, kinetics expressions of several mechanoresponsive genes were measured in apex after flame wounding or bending in order to study the long distance response at the molecular level. Results of kinetics of mechanoresponsive genes expression in apex after flame wounding (Fig. 6) showed a high increase in relative transcripts abundance in the apex for all the genes tested. Peak transcript abundance was reached as early as just 30 min after flame wounding for PtaACS6, PtaZFP2, PtaTCH2 and $1 \mathrm{~h}$ after flame wounding the basal leaf for PtaJAZ5 and PtaTCH4. For all the tested genes, expression level returned to control level only $2 \mathrm{~h}$ after flame wounding.

In contrast, results on the kinetics of mechanoresponsive gene expression after bending of the basal part of poplar stems showed no change in relative transcript abundances of any of the genes that have been studied in apices (Fig. 7). 


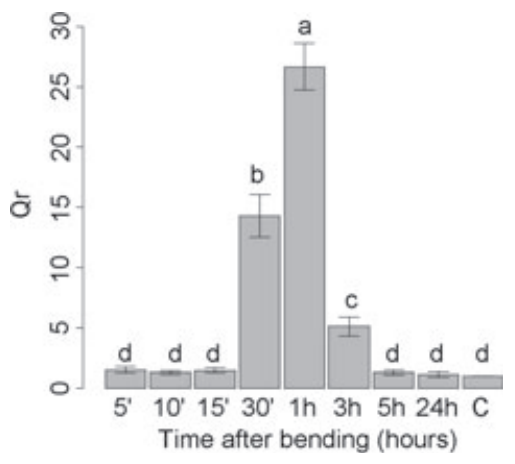

Fig. 5. Time-course accumulation of PtaJAZ5 transcripts after stem bending. Total RNA was extracted from the stem of control plants and $5,10,15,30 \mathrm{~min}$ and 1, 3, 5, 24h after mechanical stress. Relative transcript accumulation was determined by real-time quantitative PCR and calculated using the $2^{-\Delta \Delta C T}$ method with normalization from the control gene ubiquitin. Results correspond to means of six technical repetitions. Error bars represent standard error (SE). Qr values were analyzed by ANOva followed by a post hoc Tukey's HSD test to detect significant differences between Qr values. Letters indicate significantly different groups (Tukey's HSD test, $P<0.05$ ).

\section{Discussion}

These experiments were designed to reveal long-distance signaling in poplar trees through the analysis of shortterm effects of remote stimuli on both primary stem elongation and on the molecular responses in the growing tissues. Although long-term effects of mechanical stimuli on primary and secondary growth responses have already been reported in trees (Telewski and Pruyn 1998, Kern et al. 2005) and local short-term effects of bending on radial growth responses have already been studied in poplars (Coutand et al. 2009), there is still no clear picture of the short-term responses to bending of primary stem elongation in trees. In the study reported here, it was possible to analyze poplar primary growth and short-term responses to two different external stimuli. The automated non-contact experimental setup provided measurements of the primary growth at a fine scale, while minimizing the mechanical perturbation during experiments that could have generated acclimation and thus desensitization of the plants (Martin et al. 2010).

Primary stem elongation showed phasic growth with alternating nocturnal circumnutation phases and diurnal phases of growth arrest (Fig. 2). Similar circumnutationphased stem elongation patterns have been observed in A. thaliana and H. annuus (Niinuma et al. 2005, Stolarz 2009). These movements are associated with differences in turgor pressure and ionic concentration between the concave and convex faces of the stem (Niinuma et al. 2005), and could be controlled by aquaporins in order to drive the pressure variations in the elongating cells (Baskin 1986, 2007). Using mutants in A. thaliana,
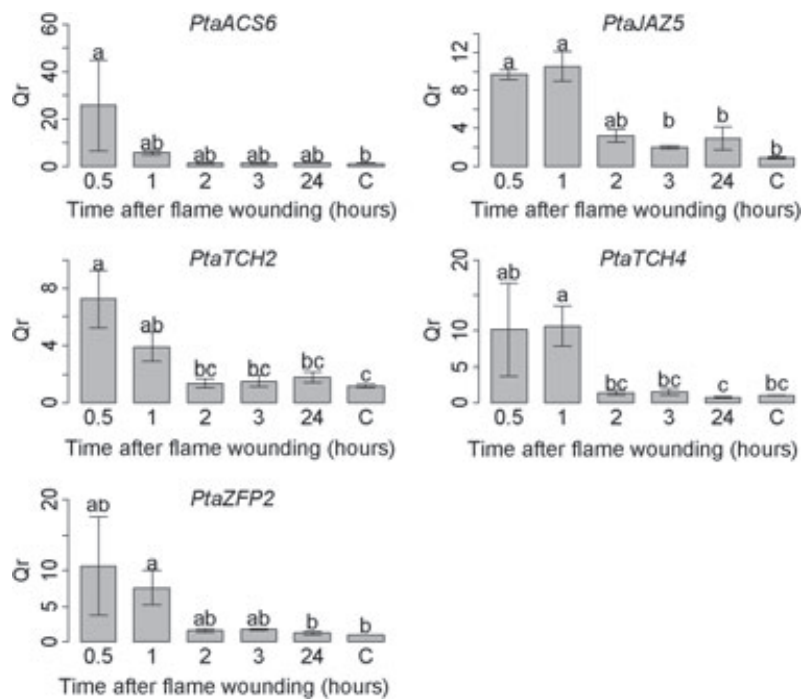

Fig. 6. Kinetics of the expression of mechanoresponsive genes in the apex after flame wounding of one basal leaf. Total RNA was extracted from the apex of control plants and 1, 2, 3, 24h after the treatment. Relative transcript accumulation was determined by realtime quantitative PCR and calculated using the $2^{-\Delta \Delta C T}$ method with normalization from the control gene ubiquitin. Results correspond to means of two independent experiments. Error bars represent standard error (SE). Qr values were analyzed by ANova followed by a post hoc Tukey's HSD test to detect significant differences between Qr values. Letters indicate significantly different groups (Tukey's HSD test, $P<0.05$ ).

Niinuma et al. (2005) showed that circumnutation is regulated by photoperiod according to a genetic control. Moreover, the modulation of circumnutation speed was regulated by a circadian clock. Schuster and Engelmann (1997) proposed that circumnutation protects the stability of the hypocotyl during elongation growth, which would otherwise be destabilized by the growth-related, simultaneous loosening of the cell wall in all the cells surrounding the organ. Besides, studies showed that circumnutation could be inhibited without suppressing growth after administration of aluminum (Hayashi et al. 2004). Finally, Stolarz (2009) suggested that cessation of circumnutation could probably be an early phase of stress response prior to inhibition of the elongation of the plant.

Poplar diurnal growth arrest phases were characterized by a very low growth rate. During this step, the plants were probably experiencing low water potential because of intense evapotranspiration, which inhibits growth (Charzewska and Zawadzki 2006). Nocturnal circumnutation phases were clearly associated with intense growth rates. At this step of the circadian cycle, plant water status was at a balance with hydroponic solution, and the low hydraulic pressure level was favorable to cell elongation. Stolarz (2009) reported in herbaceous 

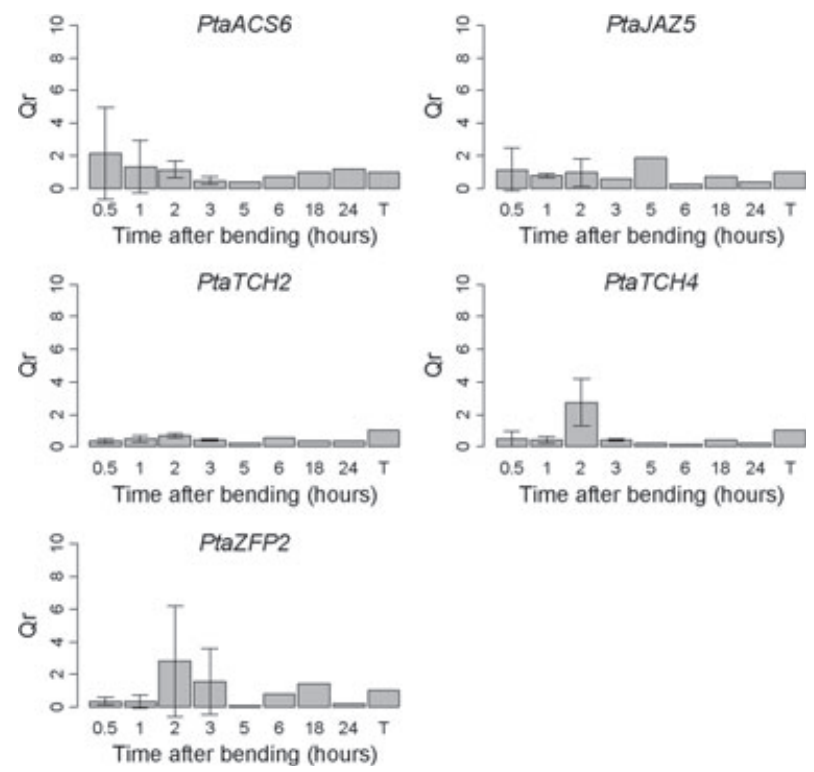

Fig. 7. Kinetics of the expression of mechanoresponsive genes in the apex after basal part of the stem bending. Total RNA was extracted from the apex of control plants and $0.5,1,2,3,5,18,24 \mathrm{~h}$ after the mechanical stimulus. Relative transcript accumulation was determined by real-time quantitative PCR and calculated using the $2^{-\Delta \Delta C T}$ method with normalization from the control gene ubiquitin. Results correspond to means of two independent experiments. Error bars represent standard error (SE). Qr values were analyzed by ANova followed by a post hoc Tukey's HSD test to detect significant differences between Qr values. No significant differences were detected.

species that circumnutation and growth rate seem to be interdependent, although their intimate relationship still remains unclear.

Non-contact growth measurements also allowed to identify growth responses to external stimuli and enlightened a significant effect of remote flame wounding to primary stem elongation, whereas no significant effect was observed after stem bending. These results were consistent with daily manual growth measurements (Fig. 3). This remote flame woundinginduced inhibition of primary growth is consistent with the literature on herbaceous species (Stahlberg and Cosgrove 1994, Stankovic and Davies 1998). Similar results were also observed on $A$. thaliana after remote wounding (Zhang and Turner 2008). Furthermore, cameras provided a high pixel density picture with short time resolution for stem elongation measurements. This experimental setup brought details about the way the primary growth was affected by the treatments among its phases. Flame wounding induced an extension of the diurnal growth arrest phase that inhibited the next nocturnal circumnutation phase and its corresponding growth rate (Fig. 4). Circumnutations were also inhibited. This is consistent with Stolarz's (2009) works who observed an intrinsic link between primary growth and circumnutation. The day after treatment, the stem elongation phases recovered their circadian rhythm; thereby highlighting that circadian rhythm was not affected in the long term. In order to compare physiological and molecular responses, the stimuli were applied at the same time for all experiments (at 10:00 h), so as to avoid any circadian rhythm-related variation in sensitivities (Garner and Bjorkman 1996). However, owing to the circadian rhythm in elongation rate, the stresses were applied at the beginning of the diurnal growth arrest phases. Thus the timing of the stresses was not optimal to characterize an inhibitory response on primary elongation. In future studies, the stimuli should be applied at the beginning of the nocturnal circumnutation phase, when growth rate level is high.

The fact that no significant primary growth responses to remote stem bending were observed can also be interpreted in another way. Indeed Pruyn et al. (2000) reported that some hybrid poplars do not present longterm primary growth responses to bending stimuli. Thus, it is possible that Populus tremula $\times$ alba clone INRA 717-1B4 lacks the thigmomorphogenetic response of primary growth to mechanical perturbations; whereas it shows local radial growth responses to stem bending (Coutand et al. 2009). This could be assessed through our analysis of genes expression.

Previously, Martin et al. (2009) reported that PtaACS6, PtaZFP2, PtaTCH2 and PtaTCH4 were all upregulated in poplar locally in the organ where the mechanical stimulus was applied. These genes are involved in several signalization pathways. PtaZFP2 is a transcription factor involved in the early stage of mechanoperception (zinc finger putative transcription factor), whereas PtaTCH2 is involved in the $\mathrm{Ca}^{2+} /$ calmodulin pathway (calmodulin-like protein). PtaTCH4 codes for a xyloglucan endotransglycosylase, which is involved in cell wall modifications, and PtaACS6 is implied in ethylene biosynthesis (acid 1-aminocyclopropane-1-carboxylate synthase). As jasmonates are involved in long-distance signaling and responses to wounding (Wasternack et al. 2006), we also investigated the expression of PtaJAZ5, which is one of the genes involved in jasmonate signaling (jasmonate ZIM-domain protein 5) and is responsive to jasmonates.

Braam and Davis (1990) reported the expression of $\mathrm{TCH}$ gene in $A$. thaliana in response to mechanical stress. Later, Martin et al. (2009) reported in poplar that PtaZFP2, PtaTCH2, PtaTCH4 genes showed local specific expression to mechanical stress in the bent segment of the stem. Here, we investigated the expression of these same genes in the apex at a remote distance from the stress application point. Ethylene and 
jasmonates have been reported as candidate markers for long-distance signaling to wounding (O'Donnell et al. 1996, Wasternack et al. 2006, Glauser et al. 2009), although Erner et al. (1980) discounted ethylene role in long distance signaling in Phaseolus vulgaris. PtaACS6 could give evidence of ethylene biosynthesis in plant's apices after remote stress, while PtaJAZ5 showed jasmonate-sensitive expression patterns.

First, PtaJAZ5 expression was assessed in local bent stem, giving evidence that the bending stimulus was sensed by the plant. This is consistent with the previous observations of Martin et al. (2009) who measured the local expressions of PtaACS6, PtaZFP2, PtaTCH2 and PtaTCH4 after a local bending of the stem with similar sum of strains. This increase in relative transcript abundance is also an evidence of jasmonate production in this region. The literature reports evidence that jasmonates are responsive to wounding (Wasternack et al. 2006, Glauser et al. 2009, Chehab et al. 2012). Thus, jasmonates seem to be involved in the regulation of local molecular responses to bending.

The kinetics of mechanoresponsive gene expression in flamed apices of basal leaf showed high induction of PtaACS6, PtaZFP2, PtaTCH2, PtaTCH4 and PtaJAZ5; suggesting that these genes were rapidly regulated in the apex after flame wounding (Fig. 6). These results suggest that both the ethylene and jasmonic acid signaling pathways are involved in long-distance signaling of flame wounding stimuli in poplar, which is consistent with previous studies assessing long-distance wound signaling in A. thaliana (Zhang and Turner 2008, Glauser et al. 2009). Recently, Heil et al. (2012) reported the strong similarities between the flame wounding stimulus and the self-recognition (leaf extract application) expression patterns in lima beans. Interestingly, flame wounding had higher similarities in terms of transcriptomic responses with self-recognition than with wounding. As the effects of the heat increase induce $d$ by flame wounding on cells are not well characterized, one should consider a potential multicomponents nature of flame wounding.

Flame wounding could induce the JA-dependent wound pathway to activate genes involved in repairing damaged tissue and activation of wound pathways (León et al. 2001).

The local time-course accumulation of PtaJAZ5 in the bent segment after bending showed the same kinetics as in the apex after flame wounding, i.e. a synchronous time-course accumulation but higher relative transcript abundance in the local bent segment of the stem (Fig. 5). Similarly, Farmer et al. 1992 observed an accumulation of systemic proteinase inhibitors in tomato leaves $20 \mathrm{~min}$ after localized wounding. Markers with earlier expression should be investigated in order to estimate the velocity of signal propagation.

Finally, molecular results were in accordance with the physiological measurements. When submitted to bending, the polar shoots from the INRA 717-1B4 clone showed neither primary growth response nor remote molecular response in the apex, despite the fact that the bending deformation has been sensed (as monitored by local gene expression). On the contrary, flame wounding inhibited primary growth by lengthening the arrest phase and induced specific mechanical molecular markers of transcript accumulation. Bending treatments were controlled to ensure the stem had experienced an elastic deformation and that it had not exceeded the elastic limit of the tissues. Vian et al. (1996) reported that a non-wounding stress (hypocotyl cooling) induced only a local calmodulin expression (no remote expression), whereas wounding induced both local and remote calmodulin expression. This is consistent with our results for bending vs flaming (Fig. 7). Furthermore, the induction of specific markers of mechanical perturbation after remote flame wounding suggests that flame wounding and bending share some signalization mechanisms, which could be explained by a mechanical components of flame wounding. On the one hand, a flame wounding stress could be responsible for cell collapse and turgor pressure loosening. On the other hand, the cell content could expand because of the heat caused by flame wounding before dying. Such expansion would generate a mechanical load on the cell wallplasma membrane-cytoskeleton complex (Baluska et al. 2003).

\section{Conclusion}

The comparison between localized flame wounding vs localized bending treatments brought first insights into poplar primary stem elongation with growth and molecular responses to remote mechanical stimuli. However, the information on primary stem elongation provided by 2D images remains incomplete, as poplar circumnutation occurs in all the lateral directions. In particular, the pendulum-like movements of the apical region in the direction perpendicular to the image plane can not be accurately evaluated and plant height was underestimated. The use of two cameras for non-contact measurement could help to estimate the real 3D position of the apex (Johnsson et al. 2009) and to provide a more relevant data set to characterize circumnutation patterns and to measure stem elongation. Furthermore, other molecular markers should be investigated. Growth markers, like genes involved in mitosis or cell elongation 
processes, would be relevant targets to better understand the growth arrest process. Protease inhibitor genes (PIN) happen to be extremely sensitive and may also be candidates as long-distance signaling markers. Recently, Beaubois et al. (2007) reported a systemic accumulation of PIN transcripts after submitting only one leaf to electromagnetic field stimulation. Such systemic accumulation of transcripts to a non-wounding stress could definitely be of interest.

Acknowledgements - The authors thank Christelle Boisselet and Sylvaine Labernia for their technical support, and Dominique Marcon for the camera settings.

\section{References}

Almeras T, Costes E, Salles JC (2004) Identification of biomechanical factors involved in stem shape variability between apricot tree varieties. Ann Bot 93: 455-468

Baluska F, Samaj J, Wojtaszek P, Volkmann D, Menzel D. (2003) Cytoskeleton-plasma membrane-cell wall continuum in plants. Emerging links revisited. Plant Physiol 133: 482-491

Baskin TI (1986) Redistribution of growth during phototropism and nutation in the pea epicotyl. Planta 169: 406-414

Baskin T (2007) Ultradian growth oscillations in organs: physiological signal or noise? In: Manusco S, Shabala S (eds) Rhythms in Plants. Phenomenology, Mechanisms, and Adaptive Significance. Springer-Verlag, Berlin-Heidelberg, pp 63-76

Beaubois E, Girard S, Lallechere S, Davies E, Paladian F, Bonnet P, Ledoigt G, Vian A (2007) Intercellular communication in plants: evidence for two rapidly transmitted systemic signals generated in response to electromagnetic field stimulation in tomato. Plant Cell Environ 30: 834-844

Braam J, Davis RW (1990) Rain-, wind-, and touch-induced expression of calmodulin and calmodulin-related genes in Arabidopsis. Cell 60: 357-364

Braam J (2005) In touch: plant responses to mechanical stimuli. New Phytol 165: 373-389

Chang S, Puryear J, Cairney J (1993) A simple and efficient method for isolating RNA from pine trees. Plant Mol Biol Rep 11: 113-116

Charzewska A, Zawadzki T (2006) Circadian modulation of circumnutation length, period, and shape in Helianthus annuus. J Plant Growth Regul 25: 324-331

Chehab W, Yao C, Henderson Z, Kim S, Braam J (2012) Arabidopsis touch-induced morphogenesis is jasmonate mediated and protects against pests. Curr Biol 22: 701-706

Christmann A, Weiler EW, Steudle E, Grill E (2007) A hydraulic signal in root-to-shoot signalling of water shortage. Plant J 52: 167-174
Coutand C, Moulia B (2000) Biomechanical study of the effect of a controlled bending on tomato stem elongation: local strain sensing and spatial integration of the signal. J Exp Bot 51: 1825-1842

Coutand C, Martin L, Leblanc-Fournier N, Decourteix M, Julien J-L, Moulia B (2009) Strain mechanosensing quantitatively controls diameter growth and PtaZFP2 gene expression in poplar. Plant Physiol 151: 223-232

Coutand C (2010) Mechanosensing and thigmomorphogenesis, a physiological and biomechanical point of view. Plant Sci 179: 168-182

Davies E (1987) Action potentials as multifunctional signals in plants: a unifying hypothesis to explain apparently disparate wound responses. Plant Cell Environ 10: 623-631

D'Errico J (2009) Shape language modelling. Available at http://www.mathworks.com/matlabcentral/fileexchange/ 24443-slm-shape-language-modeling (accessed 8 June 2010)

Dinant S (2008) Phloem, transport between organs and long-distance signalling. C R Biol 331: 334-346

Doaré O, Moulia B, De Langre E (2004) Effect of plant interaction on wind-induced crop motion. J Biomech Eng 126: 146-151

Erner Y, Biro R, Jaffe MJ (1980) Thigmomorphogenesis: Evidence for a translocatable thigmomorphogenetic factor induced by mechanical perturbation of beans (Phaseolus vulgaris). Physiol Plant 50: 21-25

Farmer EE, Johnson RR, Ryan CA (1992) Regulation of expression of proteinase-inhibitor genes by methyl jasmonate and jasmonic acid. Plant Physiol 98: 995-1002

Fromm J, Lautner S (2007) Electrical signals and their physiological significance in plants. Plant Cell Environ 30: 249-257

Garner LC, Bjorkman T (1996) Mechanical conditioning for controlling excessive elongation in tomato transplants: sensitivity to dose, frequency, and timing of brushing. J Am Soc Hortic Sci 121: 894-900

Glauser G, Dubugnon L, Mousavi SR, Rudaz S, Wolfender J-L, Farmer EE (2009) Velocity estimates for signal propagation leading to systemic jasmonic acid accumulation in wounded Arabidopsis. J Biol Chem 284: 34506-34513

Hayashi Y, Nishiyama H, Tanoi K, Ohya T, Nihei N, Tanioka K, Nakanishi TM (2004) An aluminum influence on root circumnutation in dark revealed by a new super-HARP (high-gain avalanche rushing amorphous photoconductor) camera. Plant Cell Physiol 45: $351-356$

Heil M, Ton J (2008) Long-distance signaling in plant defence. Trends Plant Sci 13: 264-272

Heil M, Ibarra-Laclette E, Adame-Álvarez RM, Martínez O, Ramirez-Chávez E, Molina-Torres J, Herrera-Estrella L (2012) How plants sense wounds: 
damaged-self recognition is based on plant-derived elicitors and induces octadecanoid signaling. PLoS One 7: e30537

Hoad GV (1995) Transport of hormones in the phloem of higher plants. Plant Growth Regul 16: 173-182

Jaeger CH, Goeschl JD, Magnuson CE, Fares Y, Strain BR (1988) Short-term responses of phloem transport to mechanical perturbation. Physiol Plant 72: 588-594

Jaffe MJ (1976) Thigmomorphogenesis - detailed characterization of the response of beans (Phaseolus vulgaris $\mathrm{L}$ ) to mechanical stimulation. Z Pflanzenphysiol 77: $437-453$

Jaffe MJ, Telewski FW, Cooke PW (1984)

Thigmomorphogenesis - on the mechanical properties of mechanically perturbed bean-plants. Physiol Plant 62: $73-78$

Jaffe MJ, Wakefield AH, Telewski F, Gulley E, Biro R, Carolina N (1985) Computer-assisted image analysis of plant growth. Plant Physiol 77: 722-730

James KR, Haritos N, Ades PK (2006) Mechanical stability of trees under dynamic loads. Am J Bot 93: 1522-1530

Julien JL, Frachisse JM (1992) Involvement of the proton pump and proton conductance change in the wave of depolarization induced by wounding in Bidens pilosa. Can J Bot 70: 1451-1458

Julien JL, Frachisse-Stoiljkovic JM (1994) Abrasion is a suitable technique for electrophysiological investigation of the slow-wave. Plant Physiol Biochem 32: 583-589

Johnsson A, Solheim BGB, Iversen T-H (2009) Gravity amplifies and microgravity decreases circumnutations in Arabidopsis thaliana stems: results from a space experiment. New Phytol 182: 621-629

Kehr J, Buhtz A (2008) Long distance transport and movement of RNA through the phloem. J Exp Bot 59: 85-92

Kern K, Ewers FW, Telewski FW, Koehler L (2005) Mechanical perturbation affects conductivity, mechanical properties and aboveground biomass of hybrid poplars. Tree Physiol 25: 1243-1251

León J, Rojo E, Sánchez-Serrano JJ (2001) Wound signalling in plants. J Exp Bot 52: 1-9

Malone M (1996) Rapid, long-distance signal transmission in higher plants. Adv Bot Res 22: 163-228

Malone M, Alarcon JJ, Palumbo L (1994) An hydraulic interpretation of rapid, long-distance wound signaling in the tomato. Planta 193: 181-185

Martin L, Leblanc-Fournier N, Azri W, Lenne C, Henry C, Coutand C, Julien JL (2009) Characterization and expression analysis under bending and other abiotic factors of PtaZFP2, a poplar gene encoding a Cys2/His2 zinc finger protein. Tree Physiol 29: 125-136

Martin L, Leblanc-Fournier N, Julien J-L, Moulia B, Coutand C (2010) Acclimation kinetics of physiological and molecular responses of plants to multiple mechanical loadings. J Exp Bot 61: 2403-2412
McMaugh S, Lyon B (2003) Real-time quantitative RT-PCR assay of gene expression in plant roots during fungal pathogenesis. Biotechniques 34: 982-986

Moulia B, Der Loughian C, Bastien R, Martin O, Rodríguez M, Gourcilleau D, Barbacci A, Badel E, Franchel G, Lenne C, Roeckel-Drevet P, Allain JM, Frachisse JM, de Langre E, Coutand C, Fournier-Leblanc N, Julien JL (2011) Integrative mechanobiology of growth and architectural development in changing mechanical environments. In: Wojtaszek P (ed) Mechanical Integration of Plant Cells and Plants. Springer, Berlin-Heidelberg, pp 269-302

Morizet J, Mingeau M (1976) Effect of environment on water-uptake, as studied on beheaded exudating tomato. 1. Role of nutrients. Ann Agron 27: 183-205

Murashige T, Skoog F (1962) A revised medium for rapid growth and bio assays with tobacco tissue cultures. Physiol Plant 15: 473-497

Neumann PM (2007) Evidence for long-distance xylem transport of signal peptide activity from tomato roots. J Exp Bot 58: 2217-2223

Niinuma K, Someya N, Kimura M, Yamaguchi I, Hamamoto $\mathrm{H}$ (2005) Circadian rhythm of circumnutation in inflorescence stems of Arabidopsis. Plant Cell Physiol 46: 1423-1427

O'Donnell P, Calvert C, Atzorn R, Wasternack C, Leyser $\mathrm{H}$, Bowles D (1996) Ethylene as a signal mediating the wound response of tomato plants. Science 274: 1914-1917

Otsu N (1979) A threshold selection method from gray-level histograms. IEEE Trans Syst Man Cybern 1: $62-66$

Pruyn ML, Ewers BJ, Telewski FW (2000)

Thigmomorphogenesis: changes in the morphology and mechanical properties of two Populus hybrids in response to mechanical perturbation. Tree Physiol 20: 535-540

Reymond P, Farmer EE (1998) Jasmonate and salicylate as global signals for defense gene expression. Curr Opin Plant Biol 1: 404-411

Reymond P, Weber H, Damond M, Farmer EE (2000) Differential gene expression in response to mechanical wounding and insect feeding in Arabidopsis. Plant Cell 12: $707-720$

Richter T, Fukshansky L (1994) Authentic in-vivo absorption-spectra for chlorophyll in leaves derived from in-situ and in-vitro measurements. Photochem Photobiol 59: 237-247

Schuster J, Engelmann W (1997) Circumnutations of Arabidopsis thaliana seedlings. Biol Rhythm Res 28: 422-444

Stahlberg R, Cleland RE, Van Volkenburgh E (2005) Decrement and amplification of slow wave potentials during their propagation in Helianthus annuus L. shoots. Planta 220: 550-558 
Stahlberg R, Cosgrove D (1994) Comparison of electric and growth responses to excision in cucumber and pea seedlings. I. Short-distance effects are a result of wounding. Plant Cell Environ 17: 43-51

Stankovic B, Davies E (1998) The wound response in tomato involves rapid growth and electrical responses, systemically up-regulated transcription of proteinase inhibitor and calmodulin and down-regulated translation. Plant Cell Physiol 39: 268-274

Stolarz M (2009) Circumnutation as a visible plant action and reaction. Plant Signal Behav 4: 380-387

Telewski FW, Jaffe MJ (1986) Thigmomorphogenesis - field and laboratory studies of Abies fraseri in response to wind or mechanical perturbation. Physiol Plant 66: $211-218$

Telewski FW, Pruyn ML (1998) Thigmomorphogenesis: a dose response to flexing in Ulmus americana seedlings. Tree Physiol 18: 65-68
Telewski FW (2006) A unified hypothesis of mechanoperception in plants. Am J Bot 93: 1466-1476

Vian A, Henry Vian C, Schantz R, Ledoigt G, Frachisse JM, Desbiez MO, Julien JL (1996) Is membrane potential involved in calmodulin gene expression after external stimulation in plants? FEBS Lett 380: 93-96

Wasternack C, Stenzel I, Hause B, Hause G, Kutter C, Maucher H, Neumerkel J, Feussner I, Miersch O (2006) The wound response in tomato - Role of jasmonic acid. J Plant Physiol 163: 297-306

Zhang Y, Turner JG (2008) Wound-induced endogenous jasmonates stunt plant growth by inhibiting mitosis. PLoS One 3: e3699

Zimmermann MR, Maischak H, Mithofer A, Boland W, Felle HH (2009) System potentials, a novel electrical long-distance apoplastic signal in plants, induced by wounding. Plant Physiol 149: 1593-1600 